\title{
The Dark Energy Camera Readout System
}

\author{
Theresa Shaw*a ${ }^{\mathrm{a}}$, Otger Ballester ${ }^{\mathrm{b}}$, Laia Cardiel-Sas ${ }^{\mathrm{b}}$, Javier Castilla ${ }^{\mathrm{c}}$, Steve Chappa ${ }^{\mathrm{a}}$, \\ Juan de Vicente ${ }^{\mathrm{c}}$, Scott Holm ${ }^{\mathrm{a}}$, Dave Huffman ${ }^{\mathrm{a}}$, Mark Kozlovsky, Gustavo Martínez ${ }^{\mathrm{c}}$, \\ Todd Moore ${ }^{\mathrm{d}}$, Jamieson Olsen ${ }^{\mathrm{a}}$, Vaidas Simaitis ${ }^{\mathrm{d}}$, Walter Stuermer ${ }^{\mathrm{a}}$ \\ ${ }^{a}$ Fermi National Accelerator Laboratory ${ }^{1}$, Box 500, Batavia, IL, USA 60510 \\ ${ }^{\mathrm{b}}$ Institut de Física d'Altes Energies (IFAE) .Edifici Cn, Universitat Autònoma de Barcelona (UAB), \\ E-08193 Bellaterra (Barcelona), Spain \\ 'Centro de Investigaciones Energéticas, Medioambientales y Tecnológias (CIEMAT), Avda. \\ Complutense, 22 - 28040 (Madrid) \\ ${ }^{\mathrm{d}}$ University of Illinois - Urbana-Champaign, Urbana, IL, USA 61801
}

\begin{abstract}
The Dark Energy Camera (DECam) was developed for use by the Dark Energy Survey (DES). The camera will be installed in the Blanco 4M telescope at the Cerro Tololo Inter-American Observatory (CTIO) and be ready for observations in the second half of 2012. The focal plane consists of $622 \times 4 \mathrm{~K}$ and $122 \times 2 \mathrm{~K}$ fully depleted CCDs. The camera provides a 3 sq. degree view and the survey will cover a 5000 sq. degree area. The camera cage and corrector have already been installed.

The development of the electronics to readout the focal plane was a collaborative effort by multiple institutions in the United States and in Spain. The goal of the electronics is to provide readout at 250 kpixels/second with less than $15 \mathrm{e}^{-}$ rms noise. Integration of these efforts and initial testing took place at Fermi National Accelerator Laboratory. DECam currently resides at CTIO and further testing has occurred in the Coudé room of the Blanco. In this paper, we describe the development of the readout system, test results and the lessons learned.
\end{abstract}

Keywords: Dark Energy, Blanco, readout, electronics, DECam

\section{INTRODUCTION}

The Dark Energy Camera (DECam) is scheduled to begin its sky survey in the late 2012. The focal plane is formed by 62 2Kx4K fully depleted Charge-Coupled Devices (CCDs). An additional 12 2kx2k CCDs are used for guiding, alignment and focus. The electronics were developed by teams operating in the US and in Spain. All integration work and final testing of DECam were done at Fermilab. A full scale telescope simulator was constructed at Fermilab, see figure 1. During February of 2011, the camera was mounted and tested at various degrees of rotation and position. Mechanical testing was also done to ensure that electronics crates could be accessed if required and noise measurements were done on a focal plane which was about two thirds populated. CTIO engineers and scientists visited Fermilab at that time to see the camera in action and get hands-on experience installing and un-installing a crate of electronics.

DECam was then removed from the telescope simulator and had its focal plane fully populated with CCDs. Testing was done in August of 2011 and everything was found to be within specifications. The camera was then carefully packed for transport and shipped to CTIO. A team of scientists and engineers then unpacked the camera and tested it in the Blanco 4M telescope Coude room in December 2011. The camera was found to be working and all CCDs responded as expected. Noise measurements on the floor of the Coude room were $\sim 7.4 \mathrm{e}^{-} \mathrm{rms}$, consistent with what we saw at Fermilab.

The camera has met and exceeded rms noise specifications. Installation on the telescope is scheduled for August 2012. This paper will describe the readout chain of the electronics and the lessons our team of engineers learned along the way.

*Tshaw@fnal.gov, 1-630-840-2478

${ }^{1}$ Operated by Universities Research Association Inc. under Contract No. DE-AC02-07CH11359 with the United States Department of Energy. 


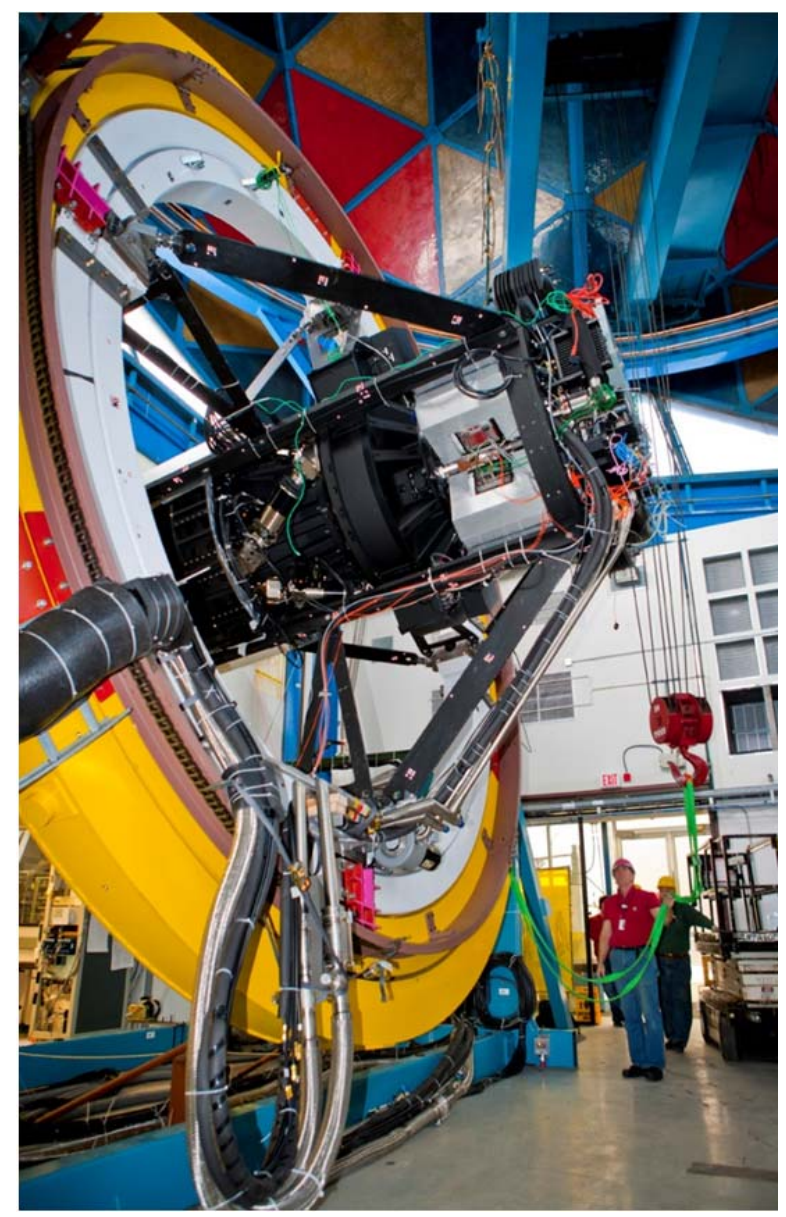

Figure 1. DECam installed on Fermilab Telescope Simulator.

\section{CCD}

\subsection{LBNL CCD}

The DES focal plane consists of sixty-two $2 \mathrm{k}$ x 4k CCDs (15 $\mu \mathrm{m}$ x $15 \mu \mathrm{m}$ pixels) arranged in a hexagon covering an imaging area of 3 sq. degrees. Smaller format $2 \mathrm{k} \mathrm{x} 2 \mathrm{k}$ CCDs are used for guiding and focus. They are located around the edges of the focal plane. We have selected the fully depleted, high-resistivity, 250 micron thick CCDs ${ }^{1}$ that have been designed and developed at the Lawrence Berkeley National Laboratory (LBNL).

The readout method we have chosen for the CCDs requires us to provide 14 clock signals and 5 bias voltages per CCD. Each CCD provides two output channels.

\subsection{CCD Output Amplifier}

The output amplifier of the LBNL CCD and many other CCDs is high impedance, calculated to be around a few thousand ohms. This presents problems when driving more than a very short cable. The way around this problem is to locate a JFET source follower near the analog output. The JFET lowers the output impedance, making it easier to drive the signal outside the dewar. This was an early lesson and we had difficulty meeting our noise specification until we incorporated this technique. 


\subsection{ESD Safe Practices}

A culture of safe ESD handling practices needed to be developed and practiced by all persons handling or working near the CCDs. This involved having people attend classes to learn ESD mitigating methods, purchasing wrist straps and ESD safe lab coats and shoes. We also learned to pay close attention to all materials we used in the lab and avoided anything which would develop a static charge. Hand held instruments provided checks on static charge build up on various materials. Humidity control was used in all labs in which CCDs were handled and humidity levels were kept at $35 \%$ or higher.

\subsection{Clock Shaping}

The wave shaping of the clocks, particularly the fast horizontal clocks, also played an important role in helping us to meet our noise specification. We found that if we did not control the rise/fall time of the clocks, we would see stronger feed through of these signals on the CCD analog outputs. The shaping was performed through the selection of amplifiers with the proper slew time and small RC filters on the clock outputs.

\subsection{Power Sequencing}

We carefully control the power sequencing of our analog supplies, both turn on and turn off. Prior to doing this, we noticed that we had spikes in bias voltages. There was some concern that this could damage the CCD, although we could not see any evidence of damage after prolonged bench testing. Nevertheless we designed a power on and power off sequence which eliminated all spikes. This is true even in the case where the AC power drops out unexpectedly. We are able to sense the loss of AC voltage in time to activate our power off sequencer.

\subsection{Full-well Capacity Damage}

At one point during the project, it was determined that we had experienced a drop of full-well capacity. We saw an average drop from around 160,000 to 120,000 electrons in some devices. It was eventually discovered that this was being caused by prolonged exposure to visible light when the CCDs had their substrate voltage turned on. Prolonged exposure to visible light is thought to move charge into the $\mathrm{SiO}_{2}$ layer where it becomes trapped. We designed an interlock system which is used to power off the readout crates, and therefore the substrate voltage, when ambient light levels are detected at the focal plane.

\subsection{Software Effects}

Another item we had to be concerned about was the software used to control the CCDs. Careful examination using an oscilloscope to look at all bias voltages and clock levels during system initialization and running allowed us to eliminate some unintentional transitions. For instance, we were able to track down some unanticipated voltage excursions to the fact that the software was re-initializing FPGAs at start up. This was not needed and we eliminated the register writes that were causing this.

\section{CCD PEDESTAL PACKAGE}

CCDs are built into pedestal packages. As shown in Figure 2, a complete pedestal package consists of the CCD, the Aluminum Nitride (AlN) readout board, the AlN spacer and the Invar foot. A 37 pin nanominiature connector is located in the center of the device and is accessed through a hole in the foot. A temporary shorting plug is inserted into the connector to provide ESD protection when the package is in storage.

The AlN readout board is approximately the same size as the CCD sensor, but slightly narrower so that the edge wirebond pads on the CCD can be used to wirebond from the CCD to the AlN backing hybrid. The thickness of the hybrid is $\sim 1 \mathrm{~mm}$ to provide mechanical stiffness and to maintain flatness. All metallization is on the top surface and is gold. Solder pads are located near the center of the hybrid for the 37-pin nanominiature series connector and for a temperature sensor.

Our early prototype packaging used flat flex connectors instead of the nanominiature connectors. We found early on that this type of connector performed poorly in a cryogenic environment and we experienced many intermittent open circuits that would go away when the system was brought back to normal temperatures. 


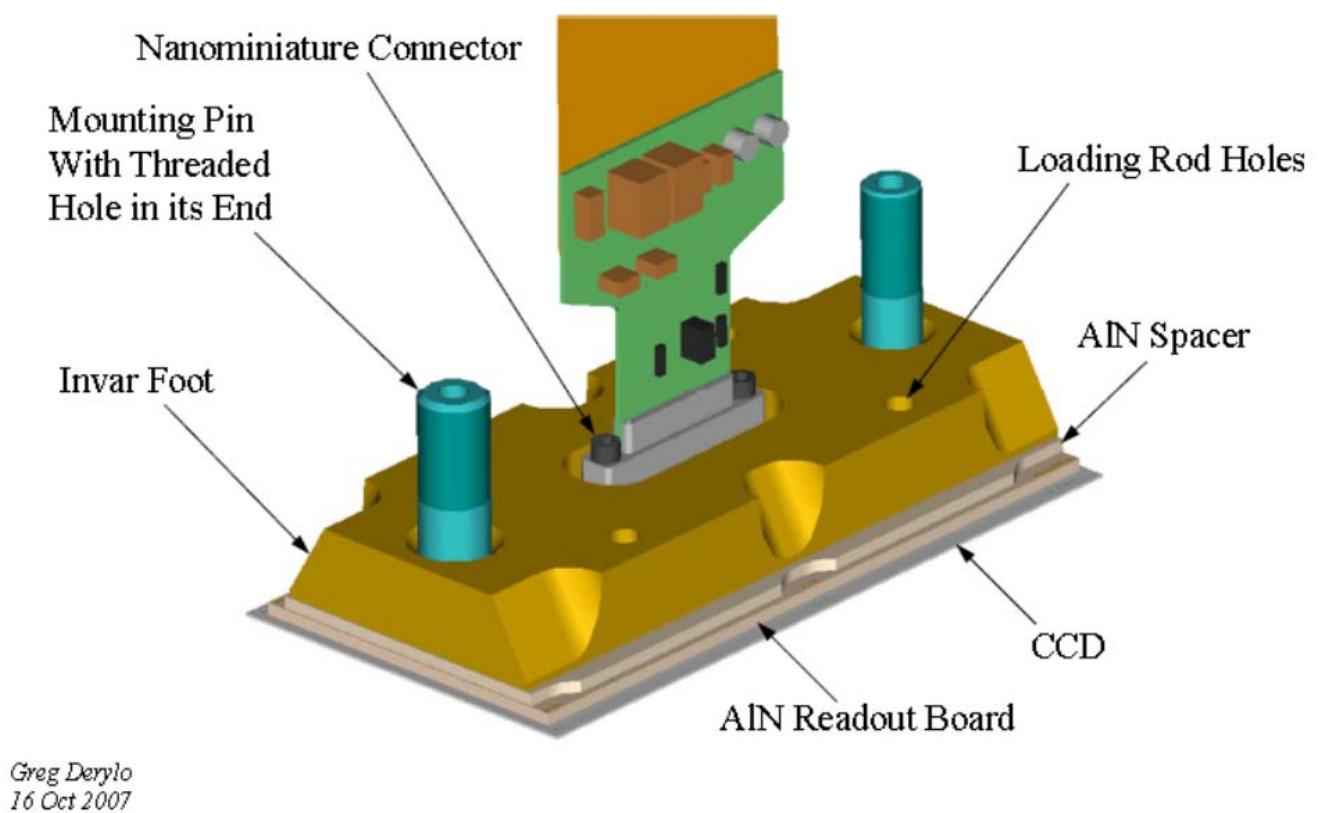

Figure 2. CCD Pedestal Package.

\section{KAPTON CABLES}

A kapton cable, shown in Figure 3, is used to carry the CCD signals between the CCD and the Vacuum Interface Board. Our kapton cable is built using a semi-rigid design. Two 8 layer printed circuit boards are built on either end of a 3-layer kapton cable. The two rigid end boards of the cable are used to plug into the Vacuum Interface Board and into the CCD pedestal package. The rigid portion of the kapton cable which plugs into the CCD pedestal package contains an active JFET source follower used to reduce the high output impedance of the CCD video output amplifier. The rigid end of the cable, which plugs into the Vacuum Interface Board, contains a pre-amplifier which drives the video signal to the Monsoon electronics. The flexible middle portion of the cable carries all signals. The cable (shields and signals) is divided into two parts, with roughly half of the cable used to carry clock signals and the other half used to carry bias voltages and the video signals. The cable is about 10 inches long, which was dictated by the mechanics of our system.

Some care had to be taken to choose the proper pre-amplifier. Our first choice suffered from unstable gain when exposed to the cold temperature of the dewar. We found a pre-amplifier which had been tested and found to work at extreme low temperatures that solved that problem.

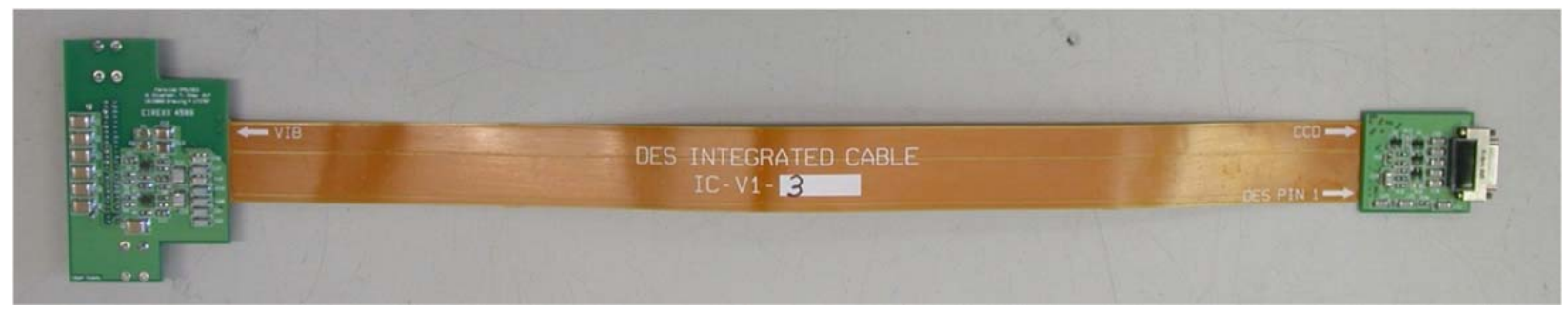

Figure 3. Semi-rigid Kapton cable. One end of the cable plugs into the Vacuum Interface Board and one end into the CCD pedestal package. 


\section{VACUUM INTERFACE BOARD}

Two Vacuum Interface Boards ${ }^{2}$ (VIBs) are used to route the clock, bias and CCD analog output signals to or from the readout electronics through the dewar wall. Once the signals are inside the dewar, they travel over Kapton cable assemblies to each CCD. The two vacuum interface boards bring a total of 2466 signals into the DECam dewar. Figure 4 is a photograph of the inside DECam. The kapton cables can be seen plugging into the VIB East and West Boards.

A great deal of care was taken in routing the signals on the VIB. Clock signals were routed carefully sandwiched between clock reference planes which were connected to the ground planes of our clock boards. Likewise, the bias voltages were sandwiched between analog reference planes which were connected to our analog readout board grounds. Substrate voltage and CCD analog outputs were routed in separate isolated paths, referenced to analog ground, to avoid noise pick-up on these sensitive signals. Vias and crossing of signals were avoided wherever possible.

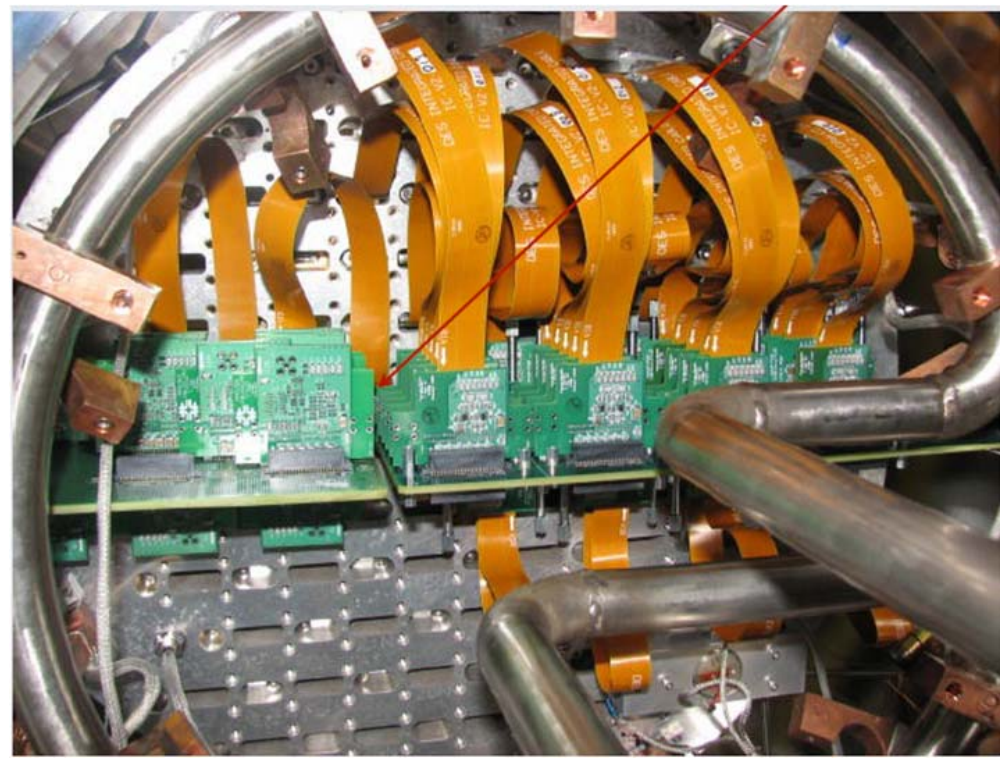

Figure 4. Inside view of the dewar showing kapton cables plugging into the VIB.

\section{EXTERNAL CABLING - READOUT TO VIB}

Two types of cabling are used to route the signals between the Readout Crates and the VIB. The Clock Board uses a shielded cable with twinax pairs. On each clock signal pair, one signal wire of the twinax carries the clock signal and one carries clock ground.

Signals going between the 12 Channel analog data acquisition module and the VIB make use of shielded coax cables. Figure 5, below, shows the external cable run implemented on our large test dewar.

\section{THE READOUT ELECTRONIC BOARDS}

The DECam Monsoon electronic boards closely follow the design of the NOAO Monsoon ${ }^{3}$ boards with some important customizations specific to DECam requirements. The Master Control Board has been modified to use an open source optical communication link, S-Link ${ }^{4}$. The 8-Channel Acquisition Board was redesigned to contain 12 channels and customized for our bias voltage needs. The Clock and Bias board was redesigned to be capable of delivering 135 clocks rather than the 32 available in the NOAO Monsoon version. These modifications were required in order for us to fit our electronics into the limited space available on the imager vessel. 


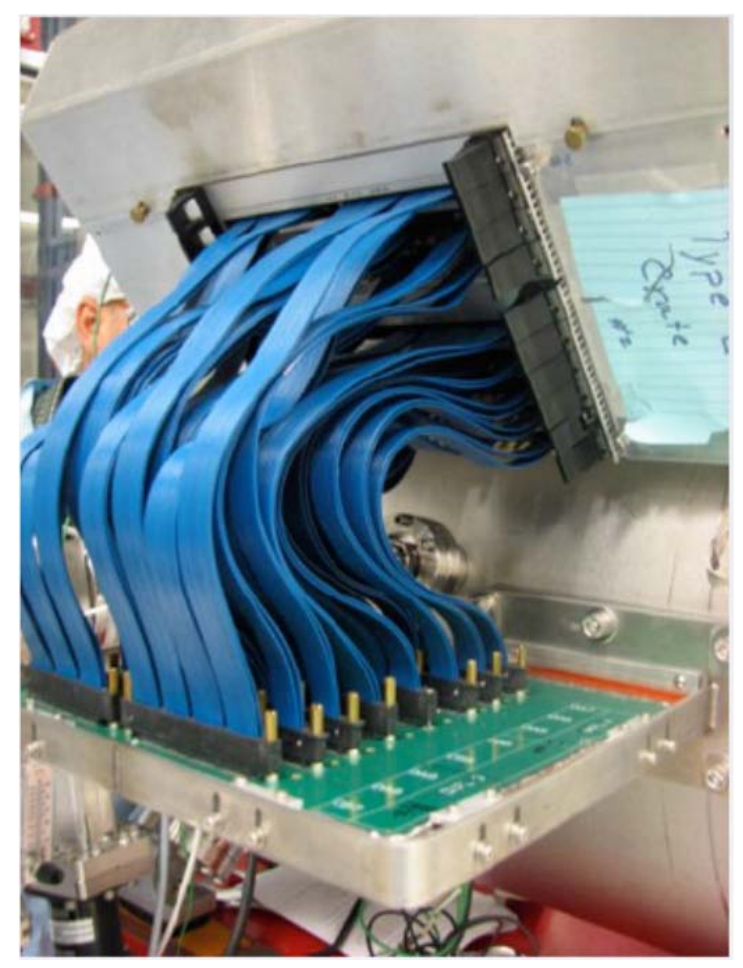

Figure 5. Photograph showing the External signal cables running between the VIB and the Monsoon Crate.

Like the original Monsoon system, all DECam Monsoon main modules have the format of a $6 \mathrm{U} 160 \mathrm{~mm} \mathrm{cPCI}^{5} \mathrm{card}$. An analog backplane has been incorporated into the crate design and all transition, or rear modules, plug directly into this analog backplane from which they draw all required analog voltages. Just as with the original Monsoon modules, a cPCI backplane is used; however most of the pin functions have been reassigned.

Figure 6 is a block diagram representing a 6-slot backplane which allows for the readout of up to 18 CCDs. This implementation makes use of a Master Control Board ${ }^{6}$, two Clock Boards ${ }^{7}$ and three 12-Channel Acquisition Boards ${ }^{8}$. DECam also makes use of a 4-slot backplane which contains a Master Control Board, one Clock Board and two 12Channel Acquisition Boards which can read out up to nine CCDs.

The DECam Readout system uses three crates, each with a 6-slot and 4-slot backplane, providing the ability to readout all 74 CCDs in the system.

\section{POWER AND GROUNDING SCHEME}

Our grounding scheme for DECam requires that we keep the camera isolated from the telescope support structure. We have little or no control over the various motors and other electrical components which are used on that structure and were aware they could present a problem for a low noise system. All camera cage mounting points are isolated through the use of G10 blocks or collars. Filtered AC power from an uninterruptable power supply is delivered directly to an AC distribution panel mounted near the camera. From there, power is distributed to all the components within our camera cage unit.

The camera dewar is also isolated from all conductive materials other than the readout crates and some control signals. Ceramic isolation is used on cryogenic cooling pipes. Through these methods we control the return paths of ground currents. 
The camera dewar is treated as the star ground central point for the readout electronics. Three front end crates are mounted to the dewar. These crates have isolated mounts to the dewar, but also have wide copper braids connected to a central grounding block on the dewar. All data to and from the crates is passed over fiber optic cables, so the front end crates and camera dewar system remains isolated from all conductive paths other than the AC power cord to each crate.

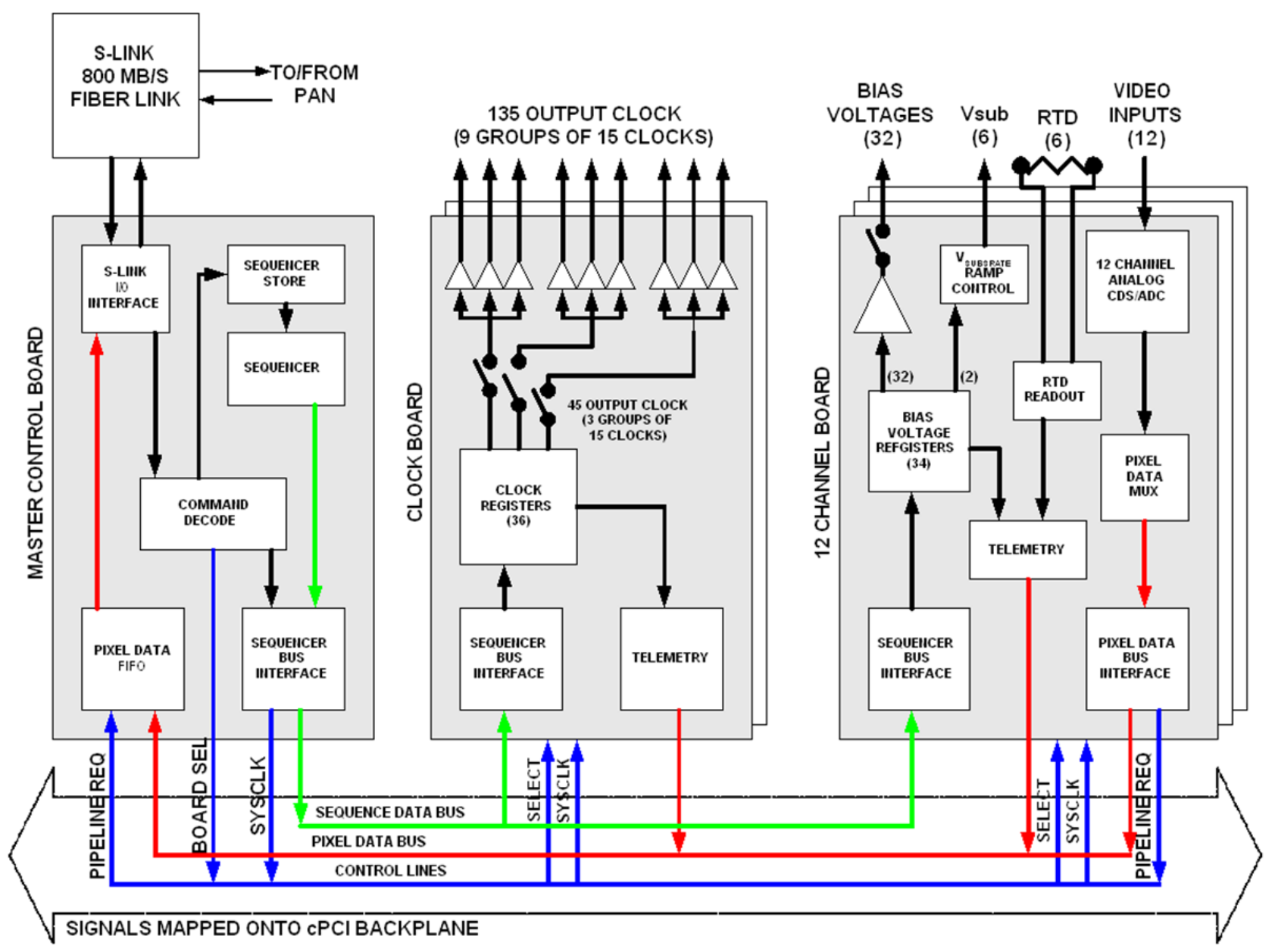

Figure 6. Block diagram of the DECam Monsoon system.

\section{LESSONS LEARNED}

The design and development of DECam represents the first time many of the DECam team of electrical engineers worked on a large scale CCD project. We all gained a lot of knowledge through the experience. The following section will summarize what has been previously described and list some of the additional lessons learned.

\subsection{CCD Lessons}

We learned the following lessons as we began to work with the LBNL p-channel CCD:

a. We had to understand the clocking patterns required to move charge efficiently through the device at the required readout rate. This involved a lot of bench testing. 
b. We had to learn how to deal with a p-channel CCD with no apparent ground reference. This caused us to think differently about ground return currents and the establishment of local reference planes.

c. We had to follow strict ESD handling methods when working with the CCDs and were not allowed to probe any pins on the device. This was a new restriction to the electronics team.

d. We came to understand that the high output impedance video driver in the CCD required an external JFET to drive signal path and lower the output impedance.

e. We learned that the CCD full well depth was reduced by exposing the CCD to ambient light while we were operating the device.

\subsection{NOAO Monsoon Readout}

We experienced the following as we worked with the selected readout architecture:

f. We had to learn how to use both the hardware and software which had been developed by NOAO when we began working with CCDs. This provided a bit of a challenge especially on the software front. It would have been helpful to have more interaction with the NOAO experts at the beginning of the project.

g. By the time electronics experts were included in the project, the Monsoon architecture had already been chosen for use as the readout system and designers were directed to follow its concepts. Looking back, from a reference/grounding point of view, we think it would have been better to re-organize the structure of the Monsoon system. Monsoon uses separate boards for the clocking and bias/video handling. We believe a better architecture would have been to design readout boards which would have provided clocks, bias voltages and video handling within individual electronics blocks/strips which would provide all services to a single CCD.

h. Clock driver selection is important. Voltage slew should be matched to requirements and RC filters used to shape the clock outputs. These were separately tuned for the fast horizontal clocks and the slower vertical clocks.

\subsection{Extreme Cold Temperatures}

Since the CCD and some of our electronics exist within the dewar and its temperature of $-100^{\circ} \mathrm{C}$ or colder, we learned the following:

i. We needed to be careful what components we selected for use inside the dewar. Our initial choice for a preamplifier was found to have unstable gain due to extreme temperature. We solved this problem by doing some document research and found an amplifier which has been tested at NASA and found to be stable at low temperatures.

j. We had a lot of problems with our initial flat flex cables and flat flex connectors. We believe the connectors had intermittent issues with various contacts opening during operations within the dewar. We solved this problem by moving to a flex cable which used more robust connectors.

\subsection{Grounding, Shielding and Power}

The following lessons were learned in the areas of grounding, shielding and power:

k. We were able to incorporate commercial switching power supplies into our system only after studying their noise and building a custom filter.

1. Given that we were dealing with a readout system which had separate clock and bias/video boards, we learned that the best low noise approach to reading out an individual CCD was to create a readout path which maintained the routing of a clock/clock_reference_plane and a bias\&video/analog_reference_plane which extended from the Monsoon readout crates to the CCD AIN boards. The planes were DC coupled at the crate backplane and AC coupled near the CCD.

$\mathrm{m}$. Power sequencing is important when turning on or off the electronics to avoid glitches on clocks/bias lines. 


\subsection{Telemetry}

Telemetry lesson include:

n. Telemetry is very important in understanding the performance of the system. We included a lot of telemetry, but due to board space restrictions can only monitor the clock voltages prior to a 1-to-3 fanout which occurs on the board. This makes us blind to the final fanout stage.

o. The Monsoon system does not include any way to test the analog channel. If space and noise considerations allowed, it would be good to build in some kind of charge injection circuit.

\section{CONCLUSION}

Electronics readout for DECam is fully instrumented. The camera has been transported from Fermilab, where all integration work was done, to CTIO where it currently sits on the floor of the Blanco 4M telescope Coude room. Noise specifications have been met and exceeded. We expect the camera to come online by the end of 2012.

\section{ACKNOWLEDGEMENTS}

Funding for the DES Projects has been provided by the U.S. Department of Energy, the U.S. National Science Foundation, the Ministry of Science and Education of Spain, the Science and Technology Facilities Council of the United Kingdom, the Higher Education Funding Council for England, the National Center for Supercomputing Applications at the University of Illinois at UrbanaChampaign, the Kavli Institute of Cosmological Physics at the University of Chicago, Financiadora de Estudos e Projetos, Fundação Carlos Chagas Filho de Amparo à Pesquisa do Estado do Rio de Janeiro, Conselho Nacional de Desenvolvimento Científico e Tecnológico and the Ministério da Ciência e Tecnologia, the German Research Foundation-sponsored cluster of excellence "Origin and Structure of the Universe” and the Collaborating Institutions in the Dark Energy Survey.

The Collaborating Institutions are Argonne National Laboratories, the University of California at Santa Cruz, the University of Cambridge, Centro de Investigaciones Energeticas, Medioambientales y Tecnologicas-Madrid, the University of Chicago, University College London, DES-Brazil, Fermilab, the University of Edinburgh, the University of Illinois at Urbana-Champaign, the Institut de Ciencies de l'Espai (IEEC/CSIC), the Institut de Fisica d'Altes Energies, the Lawrence Berkeley National Laboratory, the LudwigMaximilians Universität, the University of Michigan, the National Optical Astronomy Observatory, the University of Nottingham, the Ohio State University, the University of Pennsylvania, the University of Portsmouth, SLAC, Stanford University, and the University of Sussex.

\section{REFERENCES}

[1] Holland, S. et al. "Improved spatial resolution in thick, fully depleted CCDs with enhanced red sensitivity", IEEE Trans. Elec. Dev., 50, 225 (2003), and J. Fairfield et al., submitted to IEEE Trans. Nucl. Sci., (April 2006).

[2] Stuermer, W., “The Dark Energy Camera (DECam) Project Vacuum Interface Board Technical Design Report”, DECam 768 (February 2010).

[3] Monsoon Image Acquisition System: http://www.noao.edu/ets/new_monsoon/MNSN_DataSheet_Rev1.0.pdf

[4] CERN S-Link Homepage: http://hsi.web.cern.ch/hsi/s-link/

[5] Compact PCI Short Form Specification: http://www.picmg.org/test/pdf/cpcispec.pdf

[6] Ballester, O. and Cardiel Sas, L., “MCB Developer Notes”, DECam Internal Note 4080 (January 2010).

[7] Castilla, J., De Vincente, J. and Martinez, G., “Monsoon Clock Board”, DECam Internal Note 1215 (June 2006). 
[8] Olsen, J. and Huffman, D., "Monsoon 12-Channel Acquisition Board Firmware and Register Definitions”, DECam Internal Note 354 (May 2010). 